\title{
Imperfection Parameter Observer and Drift Compensation Controller Design of Hemispherical Resonator Gyros
}

\author{
Jaehwan $\mathrm{Pi}^{*}$ and Hyochoong Bang** \\ Department of Aerospace Engineering, Korea Advanced Institute of Science and Technology, Daejeon, Korea
}

\begin{abstract}
The hemispherical resonator gyroscope is a type of vibratory gyroscope, which can measure angle or angular rate, based on its operating mode. This paper deals with the case when the hemispherical resonator gyroscope is operated in angle measurement mode. In angle measurement mode, the resonator pattern angle precesses, with respect to the external rotation input, by the principle of the Coriolis effect, so that the external rotation can be estimated, by measuring the amount of precession angle. However, this pattern angle drifts, due to the manufacturing error of the resonator. Since the drift effect causes degradation of the angle estimation performance of the resonator, the corresponding drift compensation control should be performed, to enhance the estimation performance. In this paper, a mathematical model of the hemispherical resonator gyro is first introduced. By using the mathematical model, a nonlinear observer for imperfection parameter estimation, and the corresponding compensation controller are designed to operate hemispherical resonator gyros, as angle measurement sensors.
\end{abstract}

Key words: Hemispherical resonator gyro, Nonlinear observer, Feedback linearization

\section{Introduction}

The Hemispherical Resonator Gyroscope (HRG) is one of the vibratory gyroscopes that can be operated as an angle sensor or angular rate sensor, based on its operation mode. The vibratory gyroscope has the advantage of having no mechanically moving part, which means that it has less chance of operation failure, compared to the classical mechanical gyro. The working principle of the vibratory gyro is based on the Coriolis effect. If the external rotation of the platform occurs while the vibratory gyroscope is at the specific resonance mode termed ' $n=2$ mode', this external rotation causes precession of the resonance pattern angle. When the gyroscope is operated in angular rate measurement mode, the resonance pattern angle is controlled to have fixed value. By measuring the amount of pattern angle control input, the corresponding external angular rate can be estimated. The second mode is angle measurement mode. In this mode, the resonating pattern angle is allowed to precess freely, with respect to the external rotation. The external rotation angle can be estimated, by measuring the precession of the pattern angle.

In angle measurement mode, the pattern angle rotation is also affected by manufacturing error of the resonator, as well as the external rotation input. Although there are several other reasons, such as the temperature effect, the main reason that causes degradation of the angle estimation performance is the imperfection of the resonator. The imperfection of the resonator can be modeled with two parameters: frequency mismatch, and asymmetric damping. Frequency mismatch means the difference of the resonating frequency on two main axes. Asymmetric damping denotes the difference of the damping coefficient on two main axes.

To operate the vibratory gyros in angle measurement mode, its resonance should first be performed and maintained. Then, the quadrature vibration should be suppressed, and
This is an Open Access article distributed under the terms of the Creative Commons Attribution Non-Commercial License (http://creativecommons.org/licenses/by$\mathrm{nc} / 3.0 /$ which permits unrestricted non-commercial use, distribution, and reproduction in any medium, provided the original work is properly cited.

\footnotetext{
(c) * Ph. D Student, Corresponding author: jhpi@ascl.kaist.ac.kr ** Professor
} 
phase-locked loop should be applied [1]. The next task is to compensate the drift effect of the pattern angle, due to the imperfection parameter of the resonator. These imperfection parameters should be estimated, and correctly compensated, for accurate angle measurement.

There have been several researches on the control of the hemispherical resonator gyroscope. Lynch [1] has derived a slow-varying dynamic equation, which has the advantage that it is rather intuitive and easy to control, compared to the conventional fast-varying dynamic equation. He also designed a controller based on the slowvarying dynamics for angular rate measurement mode, by applying classical PI control law. Zuravlev [2] analyzed the drift effect of the resonating pattern angle, due to the imperfection of the resonator. Zhbanov [3] proposed a balancing mechanism to reduce the mass imbalance of the resonator, which causes the frequency mismatch of the resonator. As can be seen from the previous researches, most of the research deals with identifying and analyzing the drift effect of the resonator. However, there have been few researches to estimate the imperfection parameter, and compensate the drift effect. For the case of the MEMS (Micro-Electro-Mechanical System) gyroscope, there are several researches for compensating the drift effect of the resonator. Park $[4,5]$ applied adaptive control, based on a fast-varying dynamic equation. He also suggested applying a persistent excitation force, as well as the control force, to maintain the resonance.

In this paper, an imperfection parameter observer and corresponding compensation controller are designed for the hemispherical resonator gyroscope in angle measurement mode. The dynamic equation of the hemispherical resonator gyros is introduced, and analyzed. The imperfection parameter observer and compensation controller are designed based on the dynamic model, and are introduced. The observer for imperfection parameters is designed with nonlinear reduced order, and defining estimation error dynamics. The controller has two parts: the basic PI control, and the feedback linearization part, for compensation of the pattern angle drift effect. Finally, numerical simulation, which combines the proposed observer and controller, is performed for verification.

\section{Dynamic Model of HRG}

\subsection{Fast-Varying Model}

Before designing the observer of imperfection parameter and the controller for compensating the drift effect, a dynamic model of HRG should first be established. The dynamic model of HRG can be simplified, with its movement at the surface. The generalized CVG (Coriolis Vibratory Gyroscope) model is well known in this area [1]. A geometric representation is shown in Fig. 1.

The left side figure represents the shape of the resonator, seen from the tip. The $x$ and $y$ axes are 45 degrees separated, and the motions in each axis are independent of each other, for ideal gyros. The dynamic motions in the $\mathrm{x}$ and $\mathrm{y}$ axes are termed the generalized CVG model or fast-varying model, since the frequency of the dynamics is relatively high, compared to the slow-varying model. The right side figure denotes the slow-varying parameter, which is derived from the left side fast-varying dynamics. In the right side figure, $a$ and $q$ denote the motion of the main resonance and quadrature axis. $\theta$ is the angle between the main resonance axis, and the reference $\mathrm{x}$-axis defined by the user.

If no gravitational force and constant external rotation rate are assumed, the CVG model can be expressed as follows [1].

$$
\begin{aligned}
& \ddot{x}-2 k \Omega \dot{y}-k \Omega y+\frac{2}{\tau} \dot{x}+\Delta\left(\frac{1}{\tau}\right)\left(\dot{x} \cos 2 n \theta_{\tau}+\dot{y} \sin 2 n \theta_{\tau}\right) \\
& +\left(\omega^{2}-k^{\prime} \Omega^{2}\right) x-\omega \Delta \omega\left(x \cos 2 n \theta_{w}+y \sin 2 n \theta_{w}\right)=f_{x} \\
& \ddot{y}+2 k \Omega \dot{x}+k \Omega x+\frac{2}{\tau} \dot{y}-\Delta\left(\frac{1}{\tau}\right)\left(-\dot{x} \sin 2 n \theta_{\tau}+\dot{y} \cos 2 n \theta_{\tau}\right) \\
& +\left(\omega^{2}-k^{\prime} \Omega^{2}\right) y+\omega \Delta \omega\left(-x \sin 2 n \theta_{w}+y \cos 2 n \theta_{w}\right)=f_{y}
\end{aligned}
$$

where, $x$ and $y$ denote the main resonance axis and quadrature axis separated by 45 degrees, as shown in Figure 1. $\Omega$ represents the external angular rate input, k represents the resonator gain factor, and $f_{x}$ and $f_{y}$ denote the external acceleration input for each axis. $\omega$ and $\tau$ are the resonance frequency and damping coefficient of the HRG. $\theta_{\omega}$ and $\theta_{\tau}$ denote the orientation of the main resonance axis and main damping axis. In practice, these resonance frequencies and damping coefficients have slightly different value on each axis, such as $\omega_{1}, \omega_{2}$, and $\tau_{1}, \tau_{2}$. Their difference can be expressed as follows.

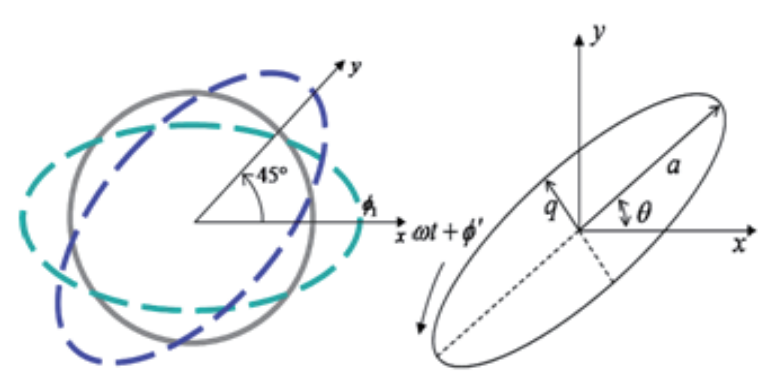

Fig. 1. Modeling of the HRG 


$$
\begin{aligned}
& \omega^{2}=\frac{\omega_{1}^{2}+\omega_{2}^{2}}{2}, \frac{1}{\tau}=\frac{1}{2}\left(\frac{1}{\tau_{1}}+\frac{1}{\tau_{2}}\right) \\
& \omega \Delta \omega=\frac{\omega_{1}^{2}-\omega_{2}^{2}}{2}, \Delta\left(\frac{1}{\tau}\right)=\frac{1}{\tau_{1}}-\frac{1}{\tau_{2}}
\end{aligned}
$$

To design a nonlinear observer for the imperfection parameter, the dynamic model should be affine, with respect to the parameter to be estimated. The fast-varying equation of motion can be rewritten as below, using the affine imperfection parameters.

$$
\begin{aligned}
& \dot{x}_{1}=x_{2} \\
& \dot{y}_{1}=y_{2} \\
& \dot{x}_{2}=-\left(\omega^{2}-\omega \omega_{c}\right) x_{1}+\omega \omega_{s} y_{1}-\left(\frac{2}{\tau}+\tau_{c}\right) x_{2}+\left(2 k \Omega-\tau_{s}\right) y_{2}+f_{x}, \\
& \dot{y}_{2}=\omega \omega_{s} x_{1}-\left(\omega^{2}+\omega \omega_{c}\right) y_{1}-\left(2 k \Omega+\tau_{s}\right) x_{2}-\left(\frac{2}{\tau}-\tau_{c}\right) y_{2}+f_{y}
\end{aligned}
$$

where, the imperfection parameters are given as below.

$$
\begin{aligned}
& \omega_{c}=\Delta \omega \cos 2 \theta_{\omega}, \omega_{s}=\Delta \omega \sin 2 \theta_{\omega}, \\
& \tau_{c}=\Delta\left(\frac{1}{\tau}\right) \cos 2 \theta_{\tau}, \tau_{s}=\Delta\left(\frac{1}{\tau}\right) \sin 2 \theta_{\tau},
\end{aligned}
$$

\subsection{Slow-Varying Model}

The fast-varying model is widely used to control the MEMS gyroscope. However, it has the disadvantage that the computational load is critical, since the control frequency should be higher than the resonance frequency. For this reason, the slow-varying model derived from the fast-varying model is widely used to control the HRG [1]. While the fast-varying model describes every instantaneous motion with respect to time, the slow-varying model describes the averaged motion of the resonating gyro, such as the resonating amplitude, the amount of quadrature vibration, and the resonating pattern angle. For this reason, the slowvarying model can reduce the computational load, and the parameter is rather intuitive, compared to the fast-varying model. The mathematical expression of the slow-varying model can be written as below [1].

$$
\begin{aligned}
\dot{E}= & =\frac{2}{\tau} E-\left(\tau_{c} \cos 2 \theta+\tau_{s} \sin 2 \theta\right) \sqrt{E^{2}-Q^{2}}-\frac{a}{\omega} f_{a s} \cos \delta \phi+\frac{q}{\omega}\left(f_{q c} \cos \delta \phi+f_{q s} \sin \delta \phi\right) \\
\dot{Q} & =-\frac{2}{\tau} Q-\left(\omega_{c} \sin 2 \theta+\omega_{s} \cos 2 \theta\right) \sqrt{E^{2}-Q^{2}}+\frac{a}{\omega}\left(f_{q c} \cos \delta \phi+f_{q s} \sin \delta \phi\right)-\frac{q}{\omega} f_{a s} \cos \delta \phi \\
\dot{\theta} & =-k \Omega+\frac{1}{2}\left(\tau_{c} \sin 2 \theta+\tau_{s} \cos 2 \theta\right) \frac{E}{\sqrt{E^{2}-Q^{2}}}+\frac{1}{2}\left(\omega_{c} \cos 2 \theta+\omega_{s} \sin 2 \theta\right) \frac{Q}{\sqrt{E^{2}-Q^{2}}} \\
& +\frac{q}{2 \omega \sqrt{E^{2}-Q^{2}}} f_{a s} \sin \delta \phi-\frac{a}{2 \omega \sqrt{E^{2}-Q^{2}}}\left(f_{q s} \cos \delta \phi-f_{q c} \sin \delta \phi\right) \\
\delta \dot{\phi}= & \dot{\phi}+\frac{1}{2}\left(\omega_{c} \cos 2 \theta+\omega_{s} \sin 2 \theta\right) \frac{E}{\sqrt{E^{2}-Q^{2}}}+\frac{1}{2}\left(\tau_{c} \sin 2 \theta-\tau_{s} \cos 2 \theta\right) \frac{Q}{\sqrt{E^{2}-Q^{2}}} \\
& +\frac{a}{2 \omega \sqrt{E^{2}-Q^{2}}} f_{a s} \sin \delta \phi-\frac{q}{2 \omega \sqrt{E^{2}-Q^{2}}}\left(f_{q s} \cos \delta \phi-f_{q c} \sin \delta \phi\right)
\end{aligned}
$$

where, $E$ is related to the resonating energy, $Q$ is related to the quadrature vibration, $\theta$ denotes the orientation of the main resonance axis, and $\delta \varphi$ denotes the phase difference between phase generator and resonator. $f_{a s}, f_{q c}, f_{q s^{\prime}}$ and $\dot{\phi}$ are the control inputs.

This paper takes advantage of the slow-varying model to design the imperfection parameter observer and corresponding compensation controller.

\section{Observer Design}

Manufacturing of the HRG always produces imperfection, such as frequency split and asymmetric damping, as previously discussed. In this chapter, a nonlinear observer is designed, to estimate the imperfection parameter.

Using eq. (5), a nonlinear observer to estimate four unknowns is designed, based on Friedland's approach [6].

The nonlinear reduced order observer has the following form.

$$
\begin{aligned}
& \hat{p}=\phi(x)+z \\
& \dot{z}=-\Phi(x) f(x, u, \hat{p})
\end{aligned}
$$

where, $x$ and $u$ are the state variable of the dynamic equation and the control input, respecively. $\hat{p}$ is the estimated unknown parameter, $\phi(x)$ is an appropriately chosen nonlinear function, and $\Phi(x)$ is its Jacobian matrix, with respect to the state variable.

For the case that the dynamics is affine with respect to the unknown parameter, the dynamics can be rewritten as below.

$$
f(x, u, p)=F(x, u) p+g(x, u)
$$

where, $F(x, u)$ is the Jacobian of the dynamic equation with respect to the known parameter, and $g(x, u)$ is the part that doesn't include the known parameter.

With the estimation error definition that $e=p-\hat{p}$, one can introduce the error dynamics, as below.

$$
\dot{e}=-\Phi(x) F(x, u) e=-L(t) e
$$

If $\Phi(x)$ is chosen such that $\mathrm{L}(\mathrm{t})$ be a positive semi-definite symmetric matrix, the estimation error converges to zero.

In this paper, two separate observers are designed, as below.

$$
p_{\tau}=\left[\begin{array}{l}
\tau_{c} \\
\tau_{s}
\end{array}\right], p_{\omega}=\left[\begin{array}{l}
\omega_{c} \\
\omega_{s}
\end{array}\right]
$$

The corresponding Jacobian matrix for the dynamic equation can be written as below. 


$$
\begin{aligned}
& F_{\omega}(x)=\frac{1}{\sqrt{E^{2}-Q^{2}}}\left[\begin{array}{cc}
0 & 0 \\
-\left(E^{2}-Q^{2}\right) \sin 2 \theta & \left(E^{2}-Q^{2}\right) \cos 2 \theta \\
\frac{Q}{2} \cos 2 \theta & \frac{Q}{2} \sin 2 \theta \\
\frac{E}{2} \cos 2 \theta & \frac{E}{2} \sin 2 \theta
\end{array}\right] \\
& F_{\tau}(x)=\frac{1}{\sqrt{E^{2}-Q^{2}}}\left[\begin{array}{cc}
-\left(E^{2}-Q^{2}\right) \cos 2 \theta & -\left(E^{2}-Q^{2}\right) \sin 2 \theta \\
0 & 0 \\
\frac{E}{2} \sin 2 \theta & -\frac{E}{2} \cos 2 \theta \\
\frac{Q}{2} \sin 2 \theta & -\frac{Q}{2} \cos 2 \theta
\end{array}\right]
\end{aligned}
$$

The nonlinear function $\square(x)$ and its Jacobian matrix $\Phi(x)$ are chosen as below.

$$
\begin{aligned}
& \phi_{\omega}=-\sqrt{E^{2}-Q^{2}}\left[\begin{array}{c}
-K_{3} \sin 2 \theta \\
K_{4} \cos 2 \theta
\end{array}\right] \\
& \phi_{\tau}=-\sqrt{E^{2}-Q^{2}}\left[\begin{array}{l}
K_{1} \cos 2 \theta \\
K_{2} \sin 2 \theta
\end{array}\right] \\
& \Phi_{\omega}(x)=\frac{1}{\sqrt{E^{2}-Q^{2}}}\left[\begin{array}{cccc}
K_{3} E \sin 2 \theta & -K_{3} Q \sin 2 \theta & 2 K_{3}\left(E^{2}-Q^{2}\right) \cos 2 \theta & 0 \\
-K_{4} E \cos 2 \theta & K_{4} Q \cos 2 \theta & 2 K_{4}\left(E^{2}-Q^{2}\right) \sin 2 \theta & 0
\end{array}\right] \\
& \Phi_{\tau}(x)=\frac{1}{\sqrt{E^{2}-Q^{2}}}\left[\begin{array}{cccc}
-K_{1} E \cos 2 \theta & K_{1} Q \cos 2 \theta & 2 K_{1}\left(E^{2}-Q^{2}\right) \sin 2 \theta & 0 \\
-K_{2} E \sin 2 \theta & K_{2} Q \sin 2 \theta & -2 K_{2}\left(E^{2}-Q^{2}\right) \cos 2 \theta & 0
\end{array}\right]
\end{aligned}
$$

where, K1, K2, K3, and K4 are constants that should be chosen appropriately for convergence.

Then, the matrix L(t) can be calculated, as follows.

$$
\begin{aligned}
& L_{\omega}=\Phi_{\omega} F_{\omega}=Q\left[\begin{array}{cc}
K_{3} & 0 \\
0 & K_{4}
\end{array}\right] \\
& L_{\tau}=\Phi_{\tau} F_{\tau}=E\left[\begin{array}{cc}
K_{1} & 0 \\
0 & K_{2}
\end{array}\right]
\end{aligned}
$$

For the conventional operation of HRG, the state variable $\mathrm{E}$ is controlled to have positive constant value for the amplitude control of the resonator, while the variable Q is controlled to be zero, for the suppression of quadrature vibration.

However, the two matrices $L_{\omega}, L_{\tau}$ should be positivedefinite, for the convergence of estimation error. As a result, the two state variable control commands for the imperfection parameter estimation stage should have the values as follow.

$$
E_{c m d}>Q_{c m d}>0
$$

\section{Controller Design}

\subsection{Basic PI Control}

The overall purpose of the HRG control loop can be divided into four purposes: 1) keep its constant resonance $(\dot{E}=0), 2)$ remove quadrature vibration $(Q=0), 3)$ remove any perturbation effect due to imperfection of the resonator $(\dot{\theta}=-k \Omega)$, and 4) track the phase of the resonance $(\delta \dot{\phi}=0)$.

Lynch [1] proposed a PI controller for the angular rate sensing mode. In a similar way, a PI controller for the angle measurement mode can be designed, as below.

$$
\begin{aligned}
& f_{a s}=K_{E}\left[E-E_{c m d}+\frac{1}{2 \tau_{E}} \int_{0}^{t}\left(E\left(t^{\prime}\right)-E_{c m d}\right) d t^{\prime}\right] \\
& f_{q c}=-K_{Q}\left[Q-Q_{c m d}+\frac{1}{2 \tau_{Q}} \int_{0}^{t}\left(Q\left(t^{\prime}\right)-Q_{c m d}\right) d t^{\prime}\right] \\
& f_{q s}=f_{q s}^{F L} \\
& \dot{\phi}=-K_{L i}\left[\operatorname{Im}(L)-L_{i c m d}+\frac{1}{2 \tau_{L i}} \int_{0}^{t}\left(\operatorname{Im}\left(L\left(t^{\prime}\right)\right)-L_{i c m d}\right) d t^{\prime}\right]
\end{aligned}
$$

In eq. (15), fas is the control command to maintain the resonance amplitude, fqc is aimed to suppress the quadrature vibration, fqs is the resonating pattern angle control, and $\dot{\phi}$ is the PLL (Phase-Locked Loop) command.

Since the angle measurement mode allows the pattern angle to precess freely with respect to the external rotation, the PI controller for pattern angle is not implemented. However, it is necessary to compensate drift effect on the pattern angle. The compensation control for pattern angle drift is proposed in the following section.

\subsection{Drift Compensation Control}

A feedback linearization method is used to cancel out the drift effect caused by imperfection of the resonator. To implement this approach, the imperfection parameter should be estimated properly, by using the parameter observer designed in the previous chapter.

The general expression for a dynamic model that includes an error parameter term can be written as below.

$$
\dot{x}=g(x)+d(x)+M(x) f
$$

where, $x, g(x), d(x), M(x)$ and $\mathrm{f}$ stand for the state vector, the dynamics excluding the error parameter term, the error term that contains the error parameter, the transformation matrix from control forces to state vector, and the control forces, respectively.

To cancel out the error term in the dynamic equation, feedback linearization control force can be implemented, as follows.

$$
\begin{aligned}
& d(x)+M(x) f_{F L}=0 \\
& f_{F L}=-M(x)^{-1} d(x)
\end{aligned}
$$


In eq. (5), all four state variables $\mathrm{E}, \mathrm{Q}, \theta, \delta \phi$ have imperfection parameters. Since the three state variables $\mathrm{E}, \mathrm{Q}$, $\delta \phi$ are controlled by the basic PI control, the only state variable that needs to be compensated is the pattern angle, $\theta$.

Since the compensation control is implemented to the pattern angle only, it is computationally efficient.

As a result, the drift compensation controller is designed as below.

$$
\begin{aligned}
d(\theta) & =\frac{1}{2}\left(\tau_{c} \sin 2 \theta+\tau_{s} \cos 2 \theta\right) \frac{E}{\sqrt{E^{2}-Q^{2}}}+\frac{1}{2}\left(\omega_{c} \cos 2 \theta+\omega_{s} \sin 2 \theta\right) \frac{Q}{\sqrt{E^{2}-Q^{2}}} \\
M(\theta) & =-\frac{a}{2 \omega \sqrt{E^{2}-Q^{2}}} \cos \delta \phi \\
f_{q s}^{F L} & =-M(\theta)^{-1} d(\theta) \\
& =\left(E\left(\tau_{c} \sin 2 \theta+\tau_{s} \cos 2 \theta\right)+Q\left(\omega_{c} \cos 2 \theta+\omega_{s} \sin 2 \theta\right)\right) \frac{\omega}{a \cos \delta \phi}
\end{aligned}
$$

\section{Simulation Result}

Numerical simulation is performed for verification of the proposed algorithm. The simulation parameters are defined

Table 1. Simulation Condition

\begin{tabular}{cccc}
\hline Parameter & Value & Parameter & Value \\
\hline$\omega$ & $7412 \mathrm{~Hz}$ & $\tau_{E}$ & 1.7 \\
$\Delta \omega$ & $1 \mathrm{~Hz}$ & $\tau_{Q}$ & 1.7 \\
$Q_{\text {factor }}$ & $10^{5}$ & $\tau_{L i}$ & 1.7 \\
$\Delta(1 / \tau)$ & $-0.02334 s^{-1}$ & $K_{E}$ & $2 \times 10^{5}$ \\
$\theta_{\omega}$ & $-5 \mathrm{deg}$. & $K_{Q}$ & $2 \times 10^{5}$ \\
$\theta_{\tau}$ & $5 \mathrm{deg}$. & $K_{L i}$ & $5 \times 10^{3}$ \\
$k$ & 0.3 & $K_{1}$ & $10^{4}$ \\
$\Omega$ & 0 & $K_{2}$ & $10^{4}$ \\
$E_{c m d}$ & 0.1 & $K_{3}$ & $10^{3}$ \\
$Q_{c m d}$ & 0.01 & $K_{4}$ & $10^{3}$ \\
\hline
\end{tabular}

as in Table 1.

The first simulation is conducted for an imperfection parameter observer. Using the imperfection parameter estimated from the observer, the second simulation is aimed to compensate the drift effect.

\subsection{Imperfection Parameter Observer}

The imperfection parameter observer simulation is conducted for 120 seconds, with three stages. The first stage for 10 seconds is to control the slow-varying state only, with the basic PI controller discussed previously. This first stage is aimed to meet the persistent excitation condition, for the convergence of estimate error. The second stage for the next 40 seconds is to estimate the frequency mismatch parameter. The final stage is to estimate the asymmetric damping parameter, by using the estimated frequency mismatch parameter.

The simulation results are shown in Figs. 2, 3, and 4.

As seen from Fig. 2, the slow-varying state variables are controlled for persistent excitation, as discussed in eq. (14). Furthermore, the pattern angle is controlled to be zero, with
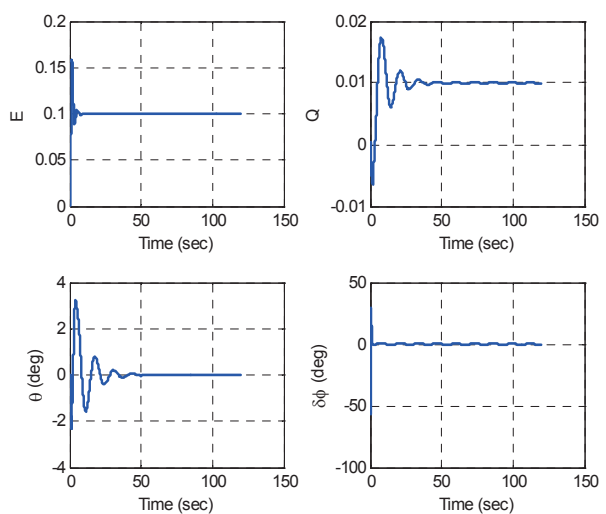

Fig. 2. Imperfection Parameter Observer - Slow-Varying Variables

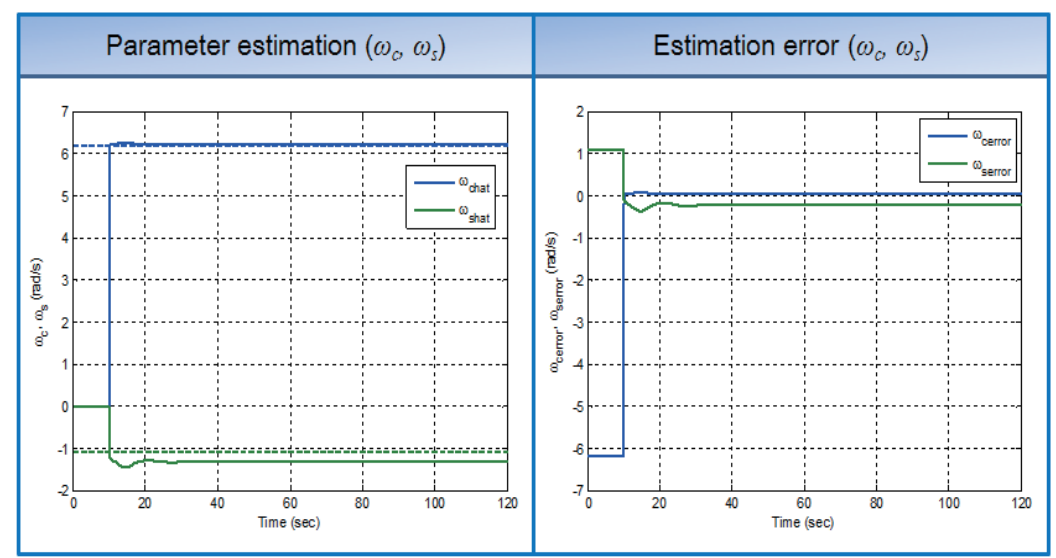

Fig. 3. Imperfection Parameter Observer - Frequency Mismatch Parameter (1) 
the basic PI control proposed by Lynch [1].

Fig. 3 shows estimation performance of the designed frequency mismatch observer. The frequency mismatch parameter observer shows satisfying performance. However, it has a slight steady-state error, since the observer uses the initial estimate value of the asymmetric damping parameter.

As seen from Fig. 4, the observer for asymmetric damping has poor estimation performance, compared to the frequency mismatch parameter observer. This error is caused by the estimation error of the frequency mismatch parameter.

If there is no estimation error on the frequency mismatch parameter, the asymmetric damping observer converges exactly, as in Fig. 5.

To check the effect of persistent excitation control, the observer for frequency mismatch is simulated, when HRG is controlled as conventional operation mode ( $\mathrm{Qcmd}=0)$. Fig. 6 shows that the observer diverges, since the persistent excitation condition is not met.

\subsection{Drift Compensation Control}

The drift compensation controller is also verified with numerical simulation. At first, numerical simulation for basic PI control without drift compensation control is performed, to analyze the drift effect. It is assumed that there is no external rotation. The result is shown in Fig. 7.

As seen from Fig. 7, the pattern angle rotates, even if there is no external rotation. Therefore, it is necessary to implement drift compensation control.

Fig. 8 shows the result when the drift compensation control is implemented with the estimated error parameter in section 5.1. The drift compensation control reduces the pattern angle drift effect, namely improving the performance of angle estimation. There still exists small drift error, caused by the small estimation error on each imperfection parameter.

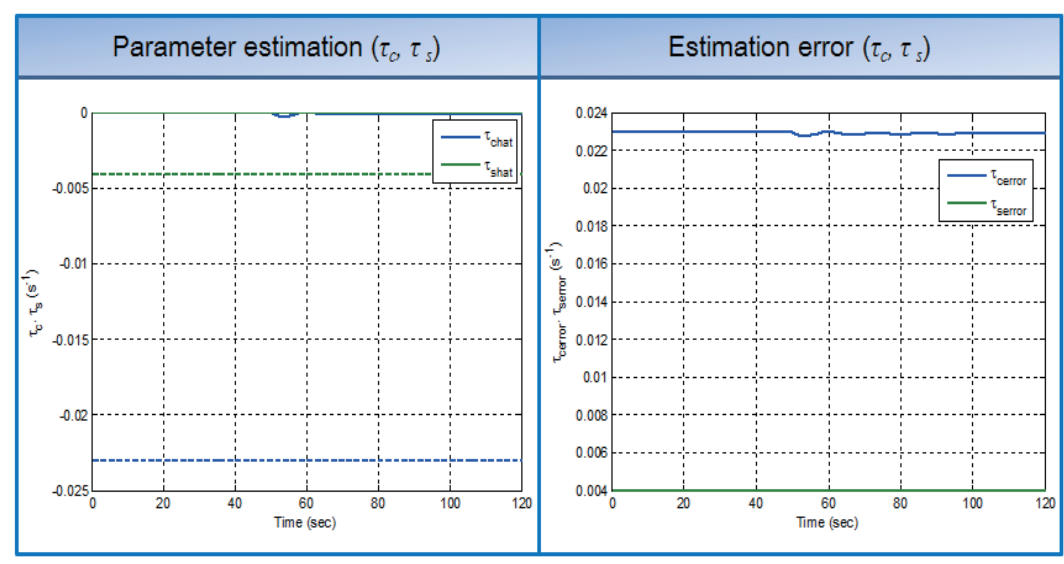

Fig. 4. Imperfection Parameter Observer - Asymmetric Damping Parameter (1)

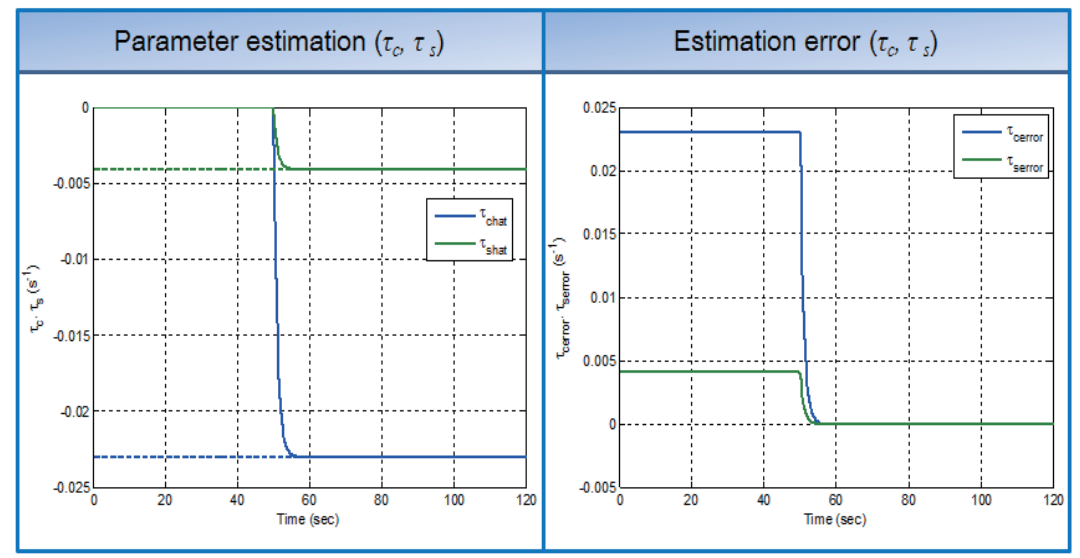

Fig. 5. Imperfection Parameter Observer - Asymmetric Damping Parameter (2) 


\section{Conclusion}

A controller for the HRG in angle measurement mode is proposed. In this mode, the pattern angle drift effect caused by imperfection of the HRG significantly affects the angle estimation performance. For this reason, drift compensation control is necessary, for its accurate operation.

The controller is composed of basic PI control and drift compensation control, using a feedback linearization method.

To implement the drift compensation controller, estimation of the imperfection parameter is essential. This estimation is performed with the proposed nonlinear observer.

Furthermore, the persistent excitation condition is studied, for the convergence of the proposed observer.

Numerical simulation is performed with the proposed control algorithm and parameter observer, for verification.
Although there is small estimation error for the imperfection parameter, the simulation results showed that the pattern angle drift effect due to the imperfection parameter is greatly reduced by the proposed imperfection parameter observer and drift compensation controller.

\section{References}

[1] D. D. Lynch, "Vibratory Gyro Analysis by the Method of Averaging", Proceedings of the 2nd International Conference on Gyroscopic Technology and Navigation, St. Petersburg, Russia, 1995.

[2] V. P. Zhuravlev, "Drift of an imperfect hemispherical resonator gyro", Mechanics of Solids, Vol. 39, No. 4, 2004, pp. 15-18.

[3] Y. K. Zhbanov, and V. P. Zhuravlev, "On the Balancing of a Hemispherical Resonator Gyro", Mechanics of Solids, Vol. 33, No. 4, 1998, pp. 2-13.

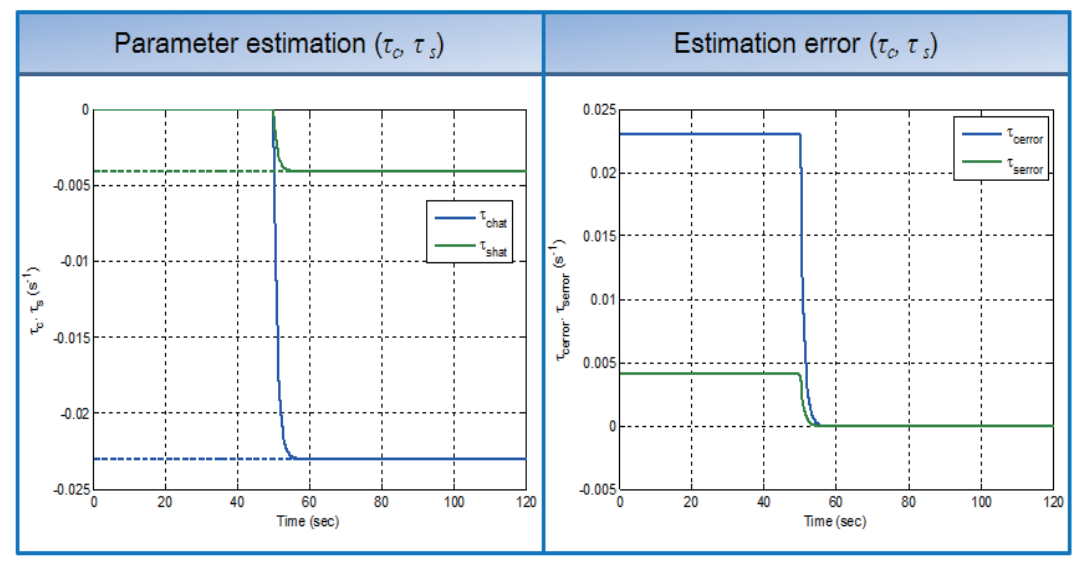

Fig. 6. Imperfection Parameter Observer - Frequency Mismatch Parameter (2)
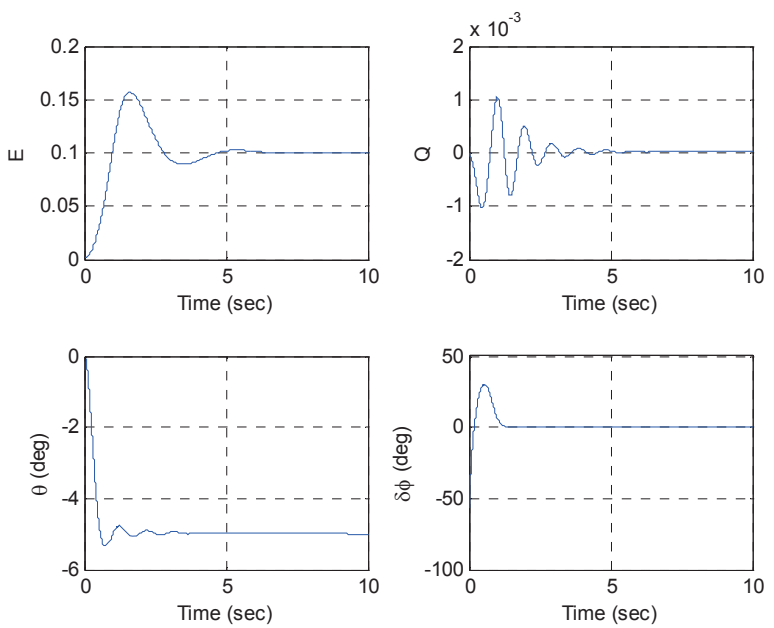

Fig. 7. Angle Measurement Mode Control - without Compensation Control
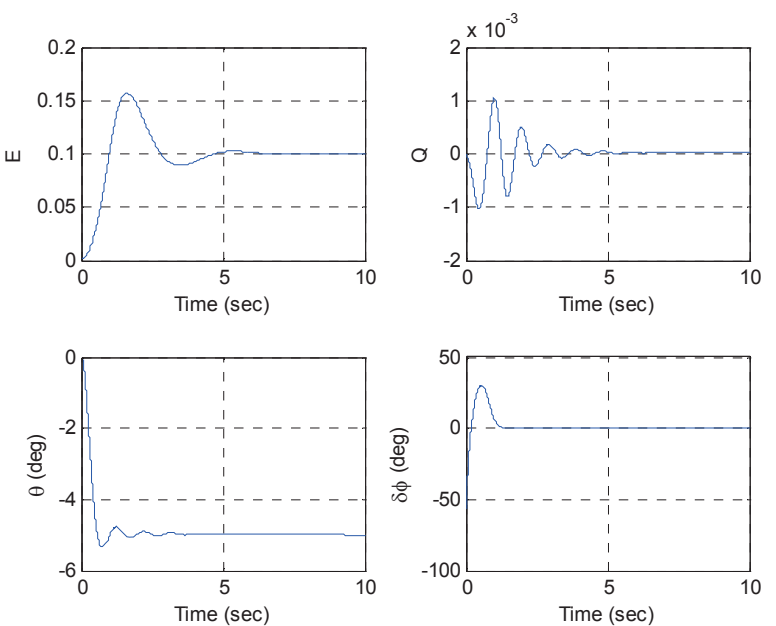

Fig. 8. Angle Measurement Mode Control - with Compensation Control 
[4] S. Park, R. Horowitz, and C. W. Tan, "Dynamics and control of a MEMS angle measuring gyroscope", Sensors and Actuator, Vol. 144, Issue 1, 2008.

[5] Sungsu Park, "Adaptive Control of a Vibratory Angle Measuring Gyroscope", Sensors, Vol. 10, No. 9, 2010, pp. 8478-
8490.

[6] Friedland B., "A Nonlinear Observer for Estimating Parameters in Dynamic Systems," Automatica, Vol.33, No. 8, 1997, pp. 1525-1530. 